\title{
Rock pulverization at extreme strain rate near the San Andreas Fault
}

\author{
Mai-Linh Doan ${ }^{* 1} \&$ Gérard Gary ${ }^{2}$
}

1 Laboratoire de Géophysique Interne et Tectonophysique - CNRS - OSUG, Université Joseph Fourier Grenoble I, BP 53, F-38041 Grenoble, France

2 Laboratoire de Mécanique des Solides, Ecole Polytechnique, Palaiseau, France

Pulverized rocks were found near the San Andreas Fault, as far as $400 \mathrm{~m}$ away from the fault core. It is exceptional to observe such a dense fragmentation so far from the core. Here we show that extreme strain rate is necessary to intensely fragment intact rocks sampled near the fault. We performed laboratory experiments on natural rocks to understand this pulverization, using a Split Hopkinson Pressure Bars apparatus. When strain rate exceeds 150/s, samples break into numerous fragments, to a scale smaller than the initial grain size. To satisfy such strain rate, we propose that the pulverization damage is associated with supershear rupture.

In the northeast of Los Angeles, the Mojave segment of the San Andreas Fault displays unusual fault damage. The whole outcrop is made of rocks finely broken to a scale smaller than the initial grain size of about $1.5 \mathrm{~mm}$. Such damage can be found as far as $400 \mathrm{~m}$ from the fault core ${ }^{1}$. This damage pattern affects mainly crystalline rocks. These rocks are not extensively weathered, with only minor clay content ${ }^{2,3}$. Another puzzling feature is the preservation of the initial isotropic grain pattern, indicating that these rocks experienced little strain ${ }^{1}$. Whereas grain comminution and gouge formation is common within the fault core, where much of the strain occurs, intense pulverization is very surprising so far from the fault core. In the damage zone around a fault, the 
strain is usually localized along fractures, leaving blocks relatively undamaged ${ }^{4,5}$. Why does this not happen near the San Andreas Fault?

The objective of this paper is to investigate the role of dynamic loading on the pulverization of rocks near the San Andreas Fault. We performed laboratory experiments on rocks sampled near the San Andreas Fault. After presenting the experiments and their results, we discuss their implication on the physics of earthquakes.

Dynamic pulverization is a process where stress localization is inhibited, so that the whole medium can be finely fractured ${ }^{6}$. Strain localization is the consequence of an unstable feedback: the largest pre-existing crack within the material is the most favourable for further fracture propagation; once extended, it becomes even more amenable to further propagation. Finally, this crack extends at the expense of the others. However, at higher loading rate, this localization process may be inhibited. The favoured crack propagates at a finite rate, at least limited by the P-wave velocity of the medium, and cannot accommodate all the energy provided to the medium. Other fractures can propagate simultaneously and coalesce to produce numerous small fragments. The sample becomes eventually pulverized ${ }^{6}$.

Did this process apply to the damaged rocks bordering the core of the San Andreas Fault? The dynamic pulverization has been considered in fault cores ${ }^{7}$, but never in the damaged zone around a fault. To quantify its plausibility, we have submitted rocks sampled near the pulverized zone to high strain rate loading. These rocks were not originally pulverized as they laid further from the fault core (about 500 m away), but they remained more damaged than the host rock. This rock material therefore reproduces the state of damage just before pulverization: not yet pulverized, but still damaged. 
The experiments were performed using a Split Hopkinson Pressure Bar (SHPB) apparatus $^{8}$ in the Laboratoire de Mécanique des Solides of the École Polytechnique, Palaiseau, France. Each sample was inserted between two bars impacted by an striker arriving at known velocity. The dimensions of the bars (3 meters long and $4 \mathrm{~cm}$ diameter) were so that the propagation of elastic waves in the bars was mainly onedimensional. Considering the analytical solution of the wave equation in the bars, forces and displacements applied to the bar extremities were retrieved. We checked that the forces were identical at the input and output bars, to verify the sample were homogenously loaded. We can then derive average stress, strain and strain rate in the sample $^{9}$ (Figure 3).

Even if the SHPB apparatus is relatively common in mechanical engineering, it is scarcely used in Earth Sciences ${ }^{10,11}$. In these experiments, we avoided two difficulties. Firstly, the lack of confining stress was not a problem, as rock pulverization $100 \mathrm{~m}$ away from the fault zone has been so far observed at the ground surface. Secondly, there was no scaling issue, as the size of the tested samples (typically $3 \mathrm{~cm}$ long with a diameter of $2.5 \mathrm{~cm}$ ) was similar to the size of the pulverized samples taken from the field. Moreover, the grain size of the rock studied here was more than ten times smaller than the sample size (Figure 1a).

Experimental results yielded three types of final states (figures 1 and 2): (1) an unbroken state, where insufficient loading did not allow to break the sample, (2) a simple fracturing state, where a sample was split by a few (at most three) longitudinal fractures - a common damage pattern for uniaxial loading at small strain rate -, and (3) a multiple fragmentation state, when the sample was ruined into multiple fragments, some with a size smaller than $1 \mathrm{~mm}$. The experiments performed at a strain rate higher than 150/s provide samples finely broken, whereas those performed below 100/s gave 
samples broken into two or three fragments (figure 2). The grain size of fragments decreased with higher strain rate.

We will now discuss the pertinence of the laboratory results to explain the natural pulverization. As the tested samples were collected in the damage zone of the San Andreas Fault, their Young modulus was small (10 $\pm 3 \mathrm{GPa}$, about one fifth of the tabulated value for granite ${ }^{12}$ ). The static strength of the material ranges between 50 and $90 \mathrm{MPa}$, about half the tabulated values for intact granite ${ }^{12}$. These low values suggest that the initial samples exhibit a dense network of cracks. All these cracks compete with the most favourable crack and help preventing strain localization along a single fracture. Consequently, we estimate that our threshold strain rate is a minimum value for the transition to fine fragmentation in the field.

Our transition does not only delimit different final damage patterns, but also corresponds to an increase in the apparent strength of the sample. These experimental results are in accordance with the statistical theory of Hild and Reynoual ${ }^{13}$ for the transition from single to multiple fracturing regimes. In both theory and experiments, rock strength starts increasing with strain rate, once in a pulverization regime.

Theoretically, there is an intrinsic increase of the material strength ${ }^{14}$, as the propagation of several small fractures requires more energy than that of a single large fracture. Experimentally, there is also a dynamic confinement phenomenon, when the sample does not instantaneously expand laterally at high strain rates. When computing the equivalent constraining stress with Forrestal formula ${ }^{15}$, we retrieve dynamic confining pressures in the range of 2 to $10 \mathrm{MPa}$, corresponding to a burial depth of only $100-500$ $\mathrm{m}$. This suggests that pulverization may also be found in the shallow subsurface.

We do not pretend to reproduce the exact state of pulverized rocks, especially its grain size distribution. The natural state is certainly the result of several earthquakes, 
each one damaging rocks by both compression and shear loading. Here, we focus only on the transition between localized fracturing and pervasive fragmentation.

We focused on dynamic pulverization to explain pulverization near faults. Other mechanisms may inhibit the strain localization process. The first one is the ductilefragile transition at high confining stress or high temperature ${ }^{16}$. But this transition is too high (300 MPa, $350^{\circ} \mathrm{C}^{16}$ ) for crystalline rocks at the ground surface. A second way to inhibit strain localization is to apply fast rotating stress. The sheared zones are then reworked over and over, so that the strain localization is annealed at the expense of the formation of preferential microstructure orientation ${ }^{17}$. This mechanism requires large strain and it is not the case for the pulverized rocks from the San Andreas Fault since they preserve their original facture.

High strain rate may be a necessary condition for pulverization, but it may not be a sufficient condition. Other phenomena can also modulate the onset of pulverization. Under tensile loading, rock strength is reduced and this may explain the asymmetry of damage observed along faults ${ }^{18}$.

There are some issues in relating the laboratory experiments to the field observations. Our experiments were performed under a uniaxial loading. The transition strain rate depends on the speed of fracture propagation and on the interaction between cracks, controlled by the statistics of the initial crack population and the size of the stress shadow zones around each crack. These parameters do not strongly depend on the fracture mode ${ }^{19}$. The fracture speed propagation is close to the $S$ wave velocity cs in all fracture modes. The shape of the stressed areas around a crack tip differs with fracture mode, but its size varies similarly, with stress decaying with distance to the fracture tip $r$ as $1 / r^{1 / 2}$. Hence, the transition from single to multiple fracturing is only weakly dependent on the fracture mode ${ }^{20}$. The strain rate condition obtained experimentally is a 
reasonable approximation to the natural case in that the sample is subject to both shear stress and normal stress.

Our results show that the transition zone is mainly related to the strain rate, rather than to the stress amplitude. Pulverized rocks are markers of events with high strain rate (>100/s). They also endured low strain amplitude, as their initial structure is preserved.

To determine in which conditions these two constraints are satisfied together, we computed the strain rate and the stress near a crack tip propagating at a constant speed. Considering distances from the fault $(r=100 \mathrm{~m})$ small relative to the rupture size (more than $10 \mathrm{~km}$ ), we calculate an asymptotic development on the distance to the rupture tip. Details of the computation are given in supplementary material. The first term of the development ${ }^{20}$ with a rupture speed below the S wave velocity (subshear rupture) yields a strain decaying as $1 / r^{1 / 2}$, and a strain rate decaying as $1 / r^{32}$. Figure 4 shows the shear strain rate of rocks located $100 \mathrm{~m}$ from the fault supposing that the maximum strain is $2 \%$, a value we think representative of the maximum strain sustained by the pulverized rocks. The maximum strain rate stays two orders of magnitude below the experimental threshold strain rate to enable pulverization. Other terms of the Taylor expansion of $\operatorname{strain}^{21}$ give similar or lower strain rate for a strain smaller than $2 \%$. Reaching the experimental threshold is therefore unlikely for subshear rupture in homogeneous material.

In the previous computation, we made two hypotheses: a homogeneous medium and a sub-shear rupture. For faults separating two different media, a sharp tensile pulse can be generated in the tensile part of the fault. If the Weertman pulse they describe induces pulverization, we expect to find pulverized rocks along bimaterial faults, with no sign of compression. However, pulverized sandstone along the San Jacinto Fault show compression features ${ }^{23}$. Moreover, pulverized rocks can be found on both sides of 
the San Andreas Fault near Lake Hughes area ${ }^{1}$. Pulverized rocks can be induced by several processes, and we propose a different mechanism for this high strain rate.

Supershear rupture induces a shock wave, with a sharp front and with small decay with distance ${ }^{20}$. High strain rates of loading can be reached $100 \mathrm{~m}$ far from the fault core as supershear rupture induces a shock wave ${ }^{24}$ and generates high frequency displacements $^{25}$. This is consistent with the discovery so far of pulverized rocks only near large strike-slip faults: San Andreas and San Jacinto Faults in California ${ }^{1,3,23}$, Northern Anatolia Fault in Turkey ${ }^{26}$, Arima-Takatsuki Fault in $\mathrm{Japan}^{27}$, which are more amenable to supershear rupture. We predict that off-fault pulverization would be only found only along large strike-slip faults, and this provides a way to test our hypothesis in the future.

This work gives constraints on the formation of pulverized rocks, which endured deformation rate larger than 100/s. As strong indicator of previous supershear rupture, moreover as an indicator independent of seismological data, pulverized rocks should be seriously considered for the risk assessment of such potentially damaging earthquakes.

\footnotetext{
Acknowledgments. We thank Raymond Barre for is laboratory help, François Hild and Kamal Safa for their comments on fracturation regimes, Michel Bouchon and Pascal Favreau for discussions on supershear rupture, Roland Leau, Robert Guiguet and Jean-Pierre Gratier for sample preparation. We appreciated the support of the direction of the LGIT and the 3S-R laboratories for their support. We thank Emily Brodsky, François Renard, Yehuda Ben-Zion and Sébastien Boutareaud for reading previous drafts of this manuscript.
}

Competing Finantial Interest Statement. Both authors have no conflict interest to disclose. 
1. Dor, O., Ben-Zion, Y., Rockwell, T.K. and Brune J.N. Pulverized Rocks in the Mojave section of the San Andreas Fault Zone, Earth Planet. Sci. Lett. 245, 642-654 (2006)

2. Wilson, B. Dewers, T. Reches, Z. and Brune, J. Particle size and energetics of gouge from earthquake rupture zones. Nature, 434,749-752 (2005)

3. Rockwell, T.K. et al. Granulometric and mineralogical properties of pulverized rocks from Tejon Pass on the San Andreas Fault and from Tejon Ranch on the Garlock Fault, California. Pure Appl. Geophys., submitted (2009).

4. Caine, J.S., Evans, J.P. and Forster, C.B. Fault zone architecture and permeability structure. Geology, 24, 1025-1028 (1996)

5. Chester, F.M., Evans, J.P. and Biegel, R.L. Internal structure and weakening mechanisms of the San Andreas fault. J. Geophys. Res. 98, 771-786 (1993)

6. Grady, D.E. and Kipp, M.E. Dynamic rock fragmentation. In Fracture Mechanics of Rocks, B.E. Atkinson Ed. Academic Press (1989)

7. Reches, Z. and Dewers, T. A. Gouge formation by dynamic pulverization during earthquake rupture. Earth. Planet. Sci. Lett. 235:361-374 (2005)

8. Kolsky. H. Stress waves in Solids. Dover Publications (1963)

9. Zhao. H. and Gary, G. On the use of SHPB techniques to determine the dynamic behavior of materials in the range of small strains. Int. J. Solids Struct., 33(23): 33633375 (1996)

10. Shan, R. , Jiang, Y. and Li, B, Obtaining dynamic complete stress-strain curves for rock using the Split Hopkinson Pressure Bar technique, Int. J. Rock. Mech. Min. Sci., 37, 983-992 (2000) 
11. Shockey, D.A., Curran, D.R., Seaman, L., Rosenberg, J.T., Petersen, C.F. Fragmentation of rock under dynamic load. Int. J. Rock. Mech. Min. Sci. 11:303-317 (1974)

12. CRC handbook of chemistry and physics: a ready-reference book of chemical and physical data (88 ${ }^{\text {th }}$ edition). Lide, D. R. ed. Boca Raton (2008).

13. Hild, F., Forquin, P. and Cordeiro da Silva, A.R. Single and multiple fragmentation of brittle geomaterials. Rev. Fr. Gen. Civ. 7(7-8):973-1003 (2003).

14. Li, Q.M. and Meng. H. About the dynamic strength enhancement of concrete-like materials in a split Hopkinson pressure bar test. Int. J. Solids Struct. 40: 343-360 (2003) 15. Forrestal, M.J., Wright, T.W. and Chen, W. The effect of radial inertia on brittle samples during the split Hopkinson pressure bar test. Int. J. Impact Eng. 34:405-411 (2007)

16. Guéguen, Y. and Palciauskas, V. Introduction to the Physics of Rocks. Princeton University Press (1994)

17. Bystricky, M., Kunze, K., Burlini, L. and Burg, J.P. High shear strain of olivine aggregate : rheological and seismic conséquences, Science, 290: 1564-1567 (2000). 18. Ben Zion, Y. and Andrews, D. J. Properties and Implication of Dynamic Rupture along a Material Interface. Bull. Seism. Soc. Am. 88(4): 1084-1094 (1998).

19. Huang, C., Subhash, G. and Vitton, S.J. A dynamic damage growth model for uniaxial compressive response of rock aggregates. Mech. Mat. 34:267-277 (2002)

20. Freund, L.B. Dynamic Fracture Mechanics. Cambridge University Press (1990) 21. Xu, Y. and Keer, L.M. Higher order asymptotic field at the moving crack tip. Int. J. Fracture. 58: 325-343, (1992).

22. Andrews, D.J. and Ben-Zion, Wrinkle-like slip pulse on a fault between different materials. J. Geophys. Res. 102: 553-571 (1995). 
23. Dor, O., Chester, J.S., Ben-Zion, Y., Brune, J.N. and Rockwell, T.K.. Damage characterization in sandstones along the Mojave section of the San Andreas Fault with a new method: implications for the depth and mechanism of rock pulverization. Pure Appl. Geoph. submitted (2009).

24. Rosakis, A., Samudrala, O. and Coker, D. Cracks faster than the shear wave speed. Science. 284(5418):1337:1340 (1999)

25. Bizarri, A. and Spudich, P. Effects of supershear rupture speed on the highfrequency content of S waves investigated using spontaneous dynamic rupture models and isochrone theory J. Geophys. Res. 113(B05304), doi:10.1029/2007JB005146 (2008) 26. Dor, O., et al. Geological and geomorphologic asymmetry across the rupture zones of the 1943 and 1944 earthquakes on the North Anatolian Fault: possible signals for preferred earthquake propagation direction. Geophys. J. Int. 173:483-504 (2008).

27. Toshi Shimamoto and Tom Mittchell, pers. comm. 
Figure 1. States of the samples after experiment. (a-left) At a low strain rate (here, 140/s), a granodiorite sample split into a few fragments when deformed in the Split Hopkinson Pressure Bar apparatus. (b-right) At a higher strain rate (here, 400/s), the sample was pulverized into numerous fragments with diameter smaller than the rock initial grain size. The ruler has centimetric marks.

Figure 2. Summary of the 27 experiments performed, where the final state relative to the peak stress and the peak strain rate are represented. The transition from single crack fracture (blue circles, figure 1 left) to intense pulverization (red stars, figure 2 right) depends on strain rate. The threshold occurs between 100/s and 150/s. Some samples remain unbroken (green squares).

Figure 3. Time-lapse snapshots of a sample being pulverized. The vertical bars lettered A, B, C and D denote the times of the above photographs, taken with four independently triggered cameras. The stress and strain history measured in our experiment is reported in the lower graphs. The sample is broken during the first loading, before the subsequent loading associated to the waves reflected at the bar extremities. The sample breaks in an early stage but because of their inertia, the fragments fly away later. The sample is fragmented while in a pure uniaxial compression, and not during stress relaxation.

Figure 4. Dilatational strain rate versus dilatational strain induced by a rupture propagating with a constant below the Rayleigh velocity in a location $100 \mathrm{~m}$ away from the fault core. As the fracture tip passes near the monitoring location strain and strain rate varies. We normalized both the strain and strain rate so that the maximum strain amplitude is $2 \%$, corresponding to the order of 
magnitude of the maximum strain experienced by the pulverized rocks near the San Andreas Fault. The curves were computed for several rupture velocities, all below the Rayleigh wave velocity (here $c_{R} \sim 0.8740301 c_{S}$ ). All curves achieve a maximum strain rate less than $0.25 / \mathrm{s}$, three orders of magnitude below the 100/s pulverisation threshold we determined in the laboratory. Details of the computation are given in supplementary material. 

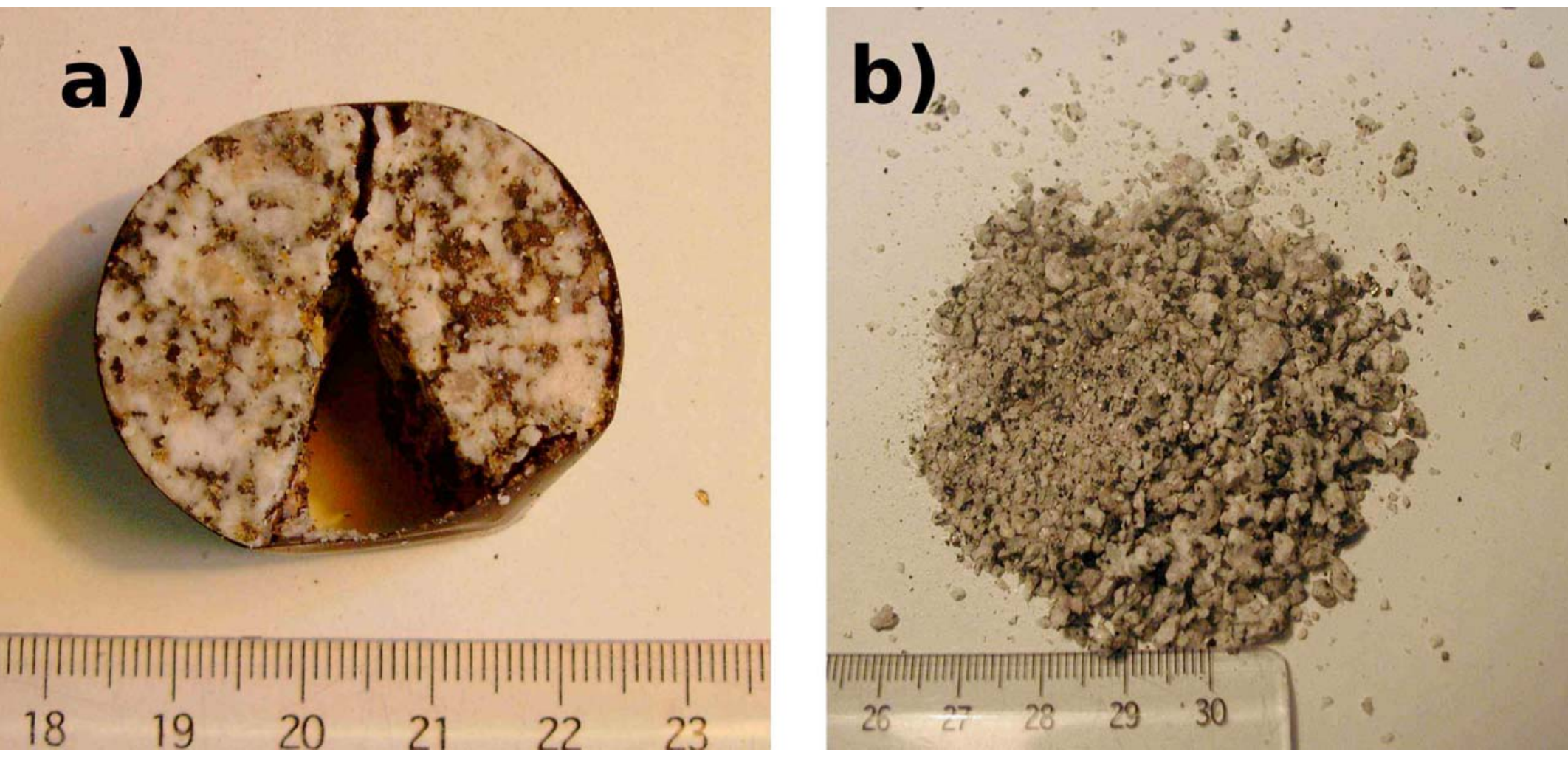
A)

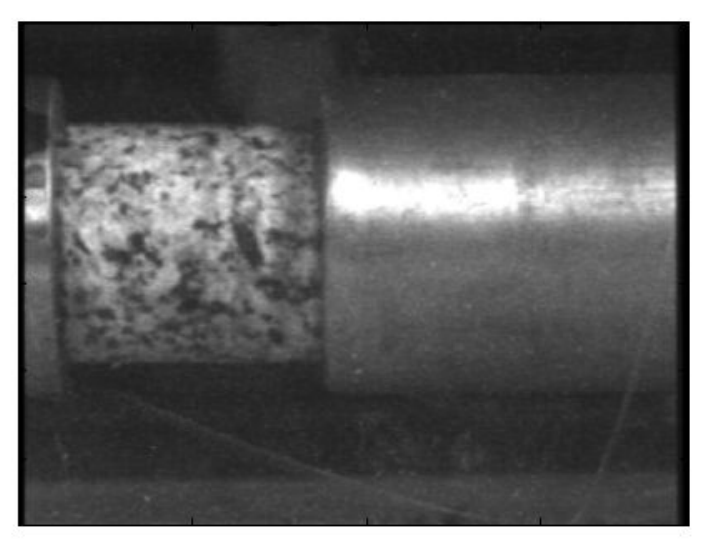

C)

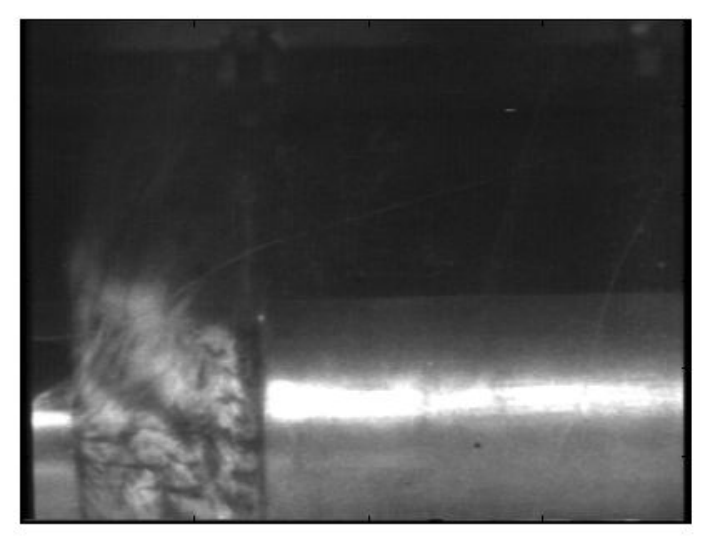

B)

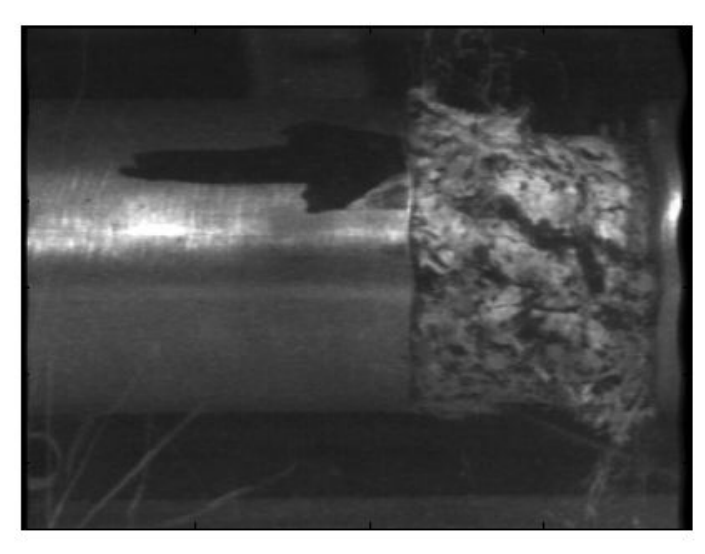

D)

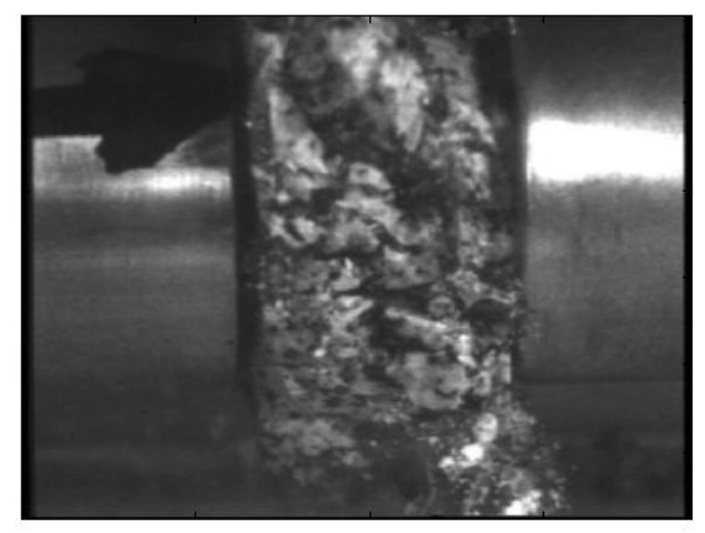

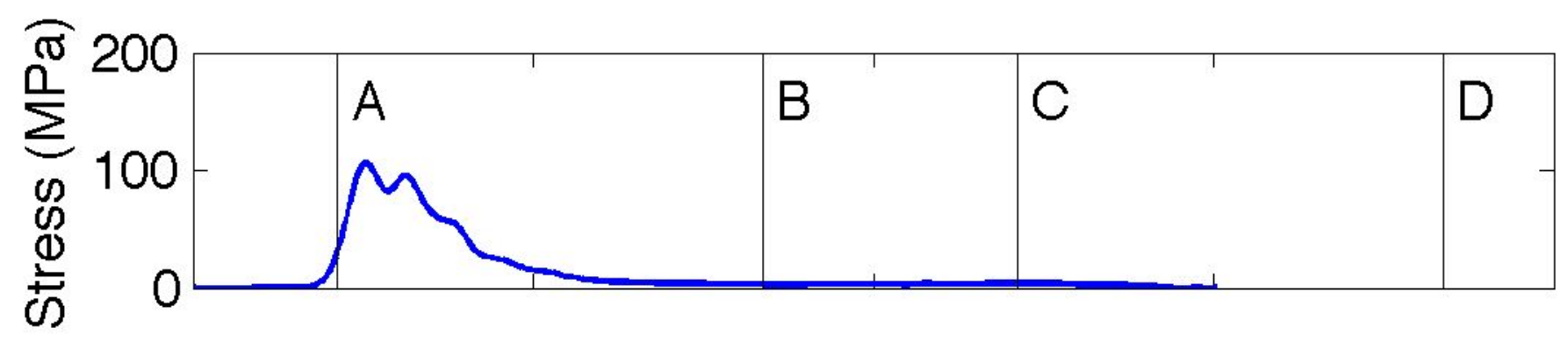

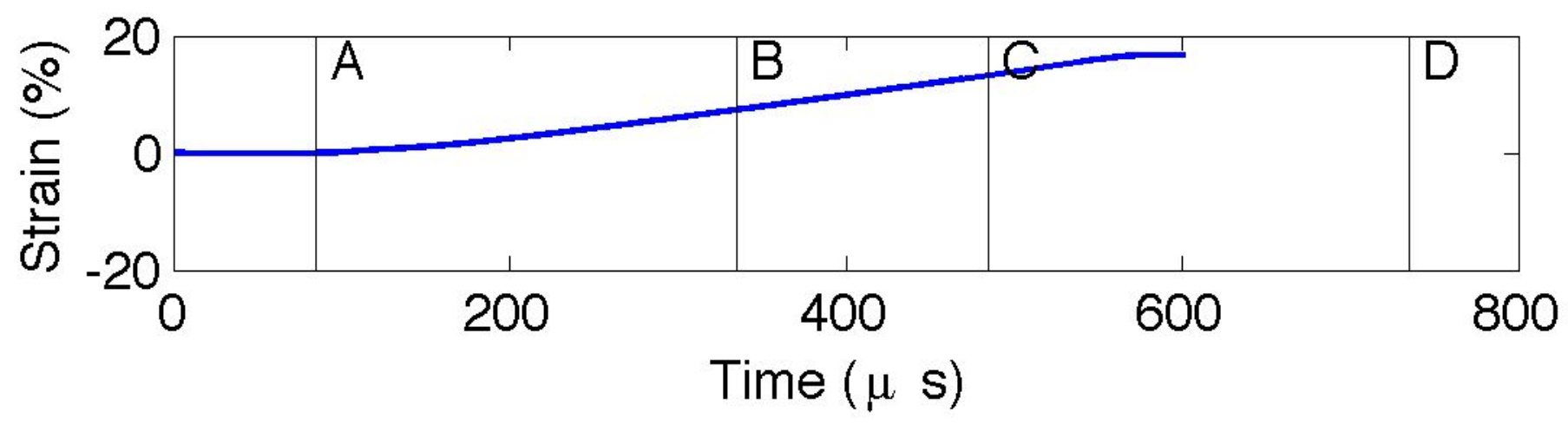




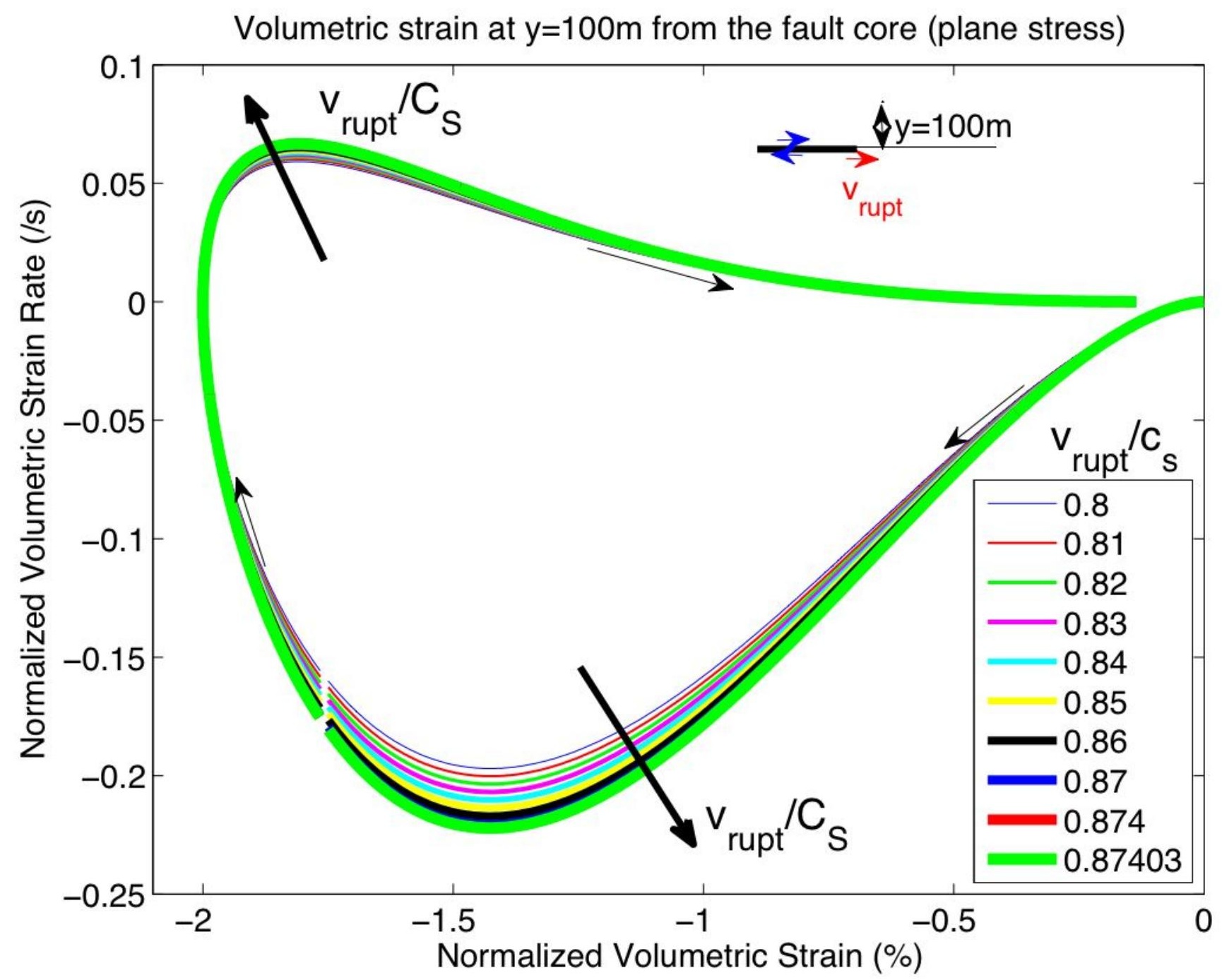

